\title{
Microwave-Assisted Synthesis of Isonitriles: A General Simple Methodology
}

\author{
Andrea Porcheddu, ${ }^{*}$ Giampaolo Giacomelli, and Margherita Salaris \\ Dipartimento di Chimica, Università degli Studi di Sassari, Via Vienna 2, I-07100 Sassari, \\ Italy \\ anpo@uniss.it
}

\section{Supporting Information}

\section{Table of Contents}

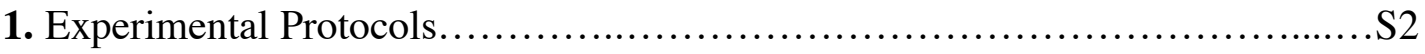

2. General Procedure: Method A............................................. 3

3. General Procedure: Method B............................................... 4

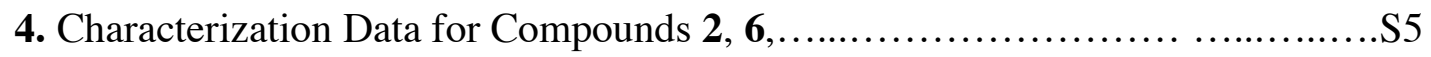

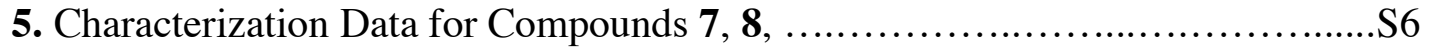

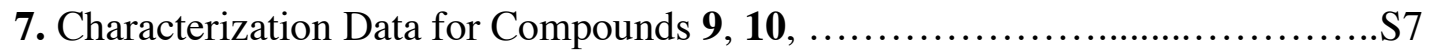

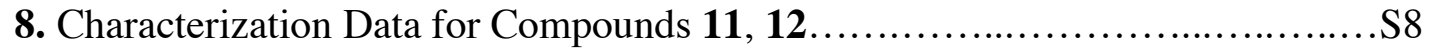

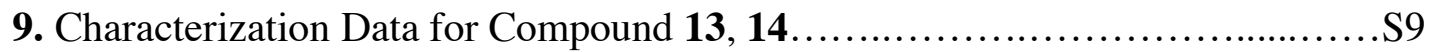

10. Characterization Data for Compound 15 ...................................S10 


\section{Experimental Protocols}

All reagents and solvents were obtained from Aldrich. All conventional reactions were run under dry nitrogen in flame-dried glassware using standard techniques unless otherwise stated. Thin-layer chromatography (TLC) analysis was performed with Merck Kieselgel 60 F254 plates and visualized using UV light, $\mathrm{KMnO}_{4}$ staining. Melting points were determined in open capillary tubes on a Buchi apparatus (Buchi B-540) and are uncorrected. The experiments were performed using using a flask equipped with a reflux condenser mounted outside the apparatus. FT-IR spectra were recorded as $\mathrm{KBr}$ pellets on Bruker Equinox 55 spectrometer (value in $\left.\mathrm{cm}^{-1}\right)$. Analytical HPLC analysis was performed with a Shimadzu analytical instrument (Shimadzu LC-2010), equipped with UV diode-array detector (Shimadzu SPD-M10AVP). Analytical HPLC was performed using a reversed phase Nucleosil

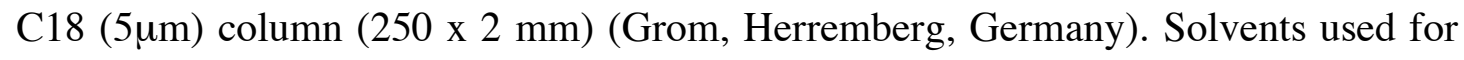
HPLC elution were (A) $\mathrm{H}_{2} \mathrm{O}$ containing $0.1 \%$ TFA, (B) methanol. Elution was performed in the isocratic condition of $20 \% \mathrm{~A}$ and $80 \% \mathrm{~B}$ over the course of $40 \mathrm{~min}$ at a flow rate of $0.8 \mathrm{~mL} / \mathrm{min}\left(220 \mathrm{~nm}\right.$ detection). ${ }^{1} \mathrm{H}$ NMR $(300 \mathrm{MHz})$ and protondecoupled ${ }^{13} \mathrm{C}$ NMR (75.4 MHz) Fourier transform spectra were obtained with Varian VXR-300 spectrometer in $\mathrm{CDCl}_{3}$ using $\mathrm{CDCl}_{3}$ solutions and TMS as an internal standard. Chemical shifts are expressed in $(\delta)$ ppm values, and coupling constants are expressed in Hertz (Hz). The following abbreviations are used: s (singlet), d (doublet), $\mathrm{m}$ (multiplet) and further qualified as a (apparent), br (broad), brs (broad singlet), c (complex). Elemental analyses were performed on a Perkin-Elmer 420 B analyzer. 
Some products, in particular, 1-isocyanopentane, tert-butylisocyanide, ${ }^{1}$ 1isocyanocyclohex-1-ene, and benzylisocyanide ${ }^{1}$ were identified by comparison with commercial products (Aldrich).

\section{General Procedure}

Method A. The procedure for the preparation of $(S)$-methyl 2-isocyano-3methylbutanoate (entry $\mathbf{9}$, Table 1 ) is representative for all aliphatic cases. In a CEM designed $10 \mathrm{~mL}$ pressure-rated reaction vial equipped with a magnetic stirrer, pyridine (120 $\mu \mathrm{L}, 1.52 \mathrm{mmol}), \mathrm{CH}_{2} \mathrm{Cl}_{2}(5.0 \mathrm{~mL})$, (S)-methyl 2-formamido-3-methylbutanoate ${ }^{2}$ $\left[0.12 \mathrm{~g}, 0.76 \mathrm{mmol},\left\{[\alpha]_{\mathrm{D}}^{25}-27.5(\mathrm{c} 1.7, \mathrm{EtOH})^{3}\right\}\right.$, and TCT, $(0.14 \mathrm{~g}, 0.76 \mathrm{mmol})$ were added sequentially at $0{ }^{\circ} \mathrm{C}$ while stirring. The vial was sealed and immediately irradiated at $100{ }^{\circ} \mathrm{C}$ (by modulation of the power) for $10 \mathrm{~min}$ in a self-tuning single mode CEM Discover ${ }^{\mathrm{TM}}$ Focused Synthesizer. The solution was cooled rapidly at room temperature by passing compressed air through the microwave cavity for $1 \mathrm{~min}$, then diluted with $\mathrm{CH}_{2} \mathrm{Cl}_{2}$ and washed with a saturated solution of $\mathrm{KHSO}_{4}$. Removal of the solvent in vacuo, gave chemically pure (S)-methyl 2-isocyano-3-methylbutanoate 9 (0.086 g, $0.61 \mathrm{mmol}, 80 \%$ yield).

(1) Walborsky, H. M.; Niznik, G. E. J. Org. Chem. 1972, 37, 187.

(2) De Luca, L.; Giacomelli, G.; Porcheddu, A.; Salaris, M. Synlett, 2004, 2570.

(3) Chancellor, T.; Morton, C. Synthesis 1994, 1023: Giard, T.; Bénard, D.; Plaquevent, J. C. Synthesis 1998, 297. 
Method B. The preparation of 1-isocyano-4-methoxybenzene is representative for the aromatic isonitriles. To $N$-(4-methoxyphenyl)formamide $(0.08 \mathrm{~g}, 0.53 \mathrm{mmol})$ contained in a CEM $10 \mathrm{~mL}$ pressure-rated vial equipped with a magnetic stirrer, TEA (240 $\mu \mathrm{L}, 1.75 \mathrm{mmol})$, TCT $(0.29 \mathrm{~g}, 1.59 \mathrm{mmol})$, and $\mathrm{CH}_{2} \mathrm{Cl}_{2}(5.0 \mathrm{~mL})$ were added sequentially at $0^{\circ} \mathrm{C}$. The vial was sealed, immediately irradiated at $50{ }^{\circ} \mathrm{C}$ (by modulation of the power) for $3 \mathrm{~min}$. The solution was cooled rapidly at room temperature by passing compressed air through the microwave cavity for $1 \mathrm{~min}$, then diluted with $\mathrm{CH}_{2} \mathrm{Cl}_{2}$ and washed with a saturated solution of $\mathrm{KHSO}_{4}$. Removal of the solvent under vacuum, gave $0.053 \mathrm{~g}$ of chemically pure 1-isocyano-4methoxybenzene 12 (75\% yield). ${ }^{4}$

(4) Hammick, D. L.; New, R. C. A.; Sidgwick, N. V.; Sutton, L. E. J. Chem, Soc 1930, 1876. 


\section{Characterization Data}

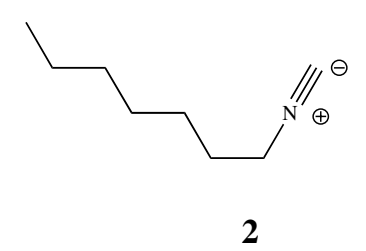

2: $80 \%$ yield. ${ }^{1} \mathrm{H}$ NMR $\left(\mathrm{CDCl}_{3}\right): \delta(\mathrm{ppm}) 3.48-3.40(\mathrm{~m}, 1 \mathrm{H}), 3.30-3.23(\mathrm{~m}, 1 \mathrm{H}), 1.59-$ $1.53(\mathrm{~m}, 2 \mathrm{H}), 1.30-1.22(\mathrm{~m}, 8 \mathrm{H}), 0.86(\mathrm{~m}, 3 \mathrm{H}) .{ }^{13} \mathrm{C} \mathrm{NMR}\left(\mathrm{CDCl}_{3}\right): \delta(\mathrm{ppm})$ 161.2, 41.0, 31.5, 29.4, 28.8, 26.7, 22.4, 13.8. Anal. Calcd for $\mathrm{C}_{8} \mathrm{H}_{15} \mathrm{~N}$ : C, 76.74; H, 12.07; N, 11.19. Found: C, 76.71; H, 12.19; N, 11.30.

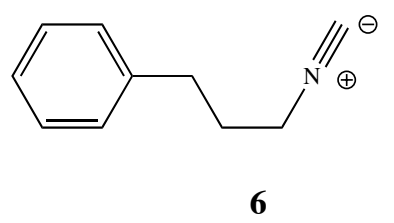

6: $95 \%$ yield. IR 3061, 3026, 2929, 2859, 2148 (NC), 1663, 1539, 747. ${ }^{1} \mathrm{H}$ NMR $\left(\mathrm{CDCl}_{3}\right): \delta(\mathrm{ppm}) 7.28-7.13(\mathrm{~m}, 5 \mathrm{H}), 3.31-3.24(\mathrm{~m}, 1 \mathrm{H}), 3.19-3.13(\mathrm{~m}, 1 \mathrm{H}), 2.66-2.59$ $(\mathrm{m}, 2 \mathrm{H}), 1.87-1.80(\mathrm{~m}, 2 \mathrm{H}) .{ }^{13} \mathrm{C} \mathrm{NMR}\left(\mathrm{CDCl}_{3}\right): \delta(\mathrm{ppm}) 161.43,141.08,128.4,128.2$, 125.9, 37.6, 32.9, 30.9. (ESI + ve ion). Calcd for $\mathrm{C}_{10} \mathrm{H}_{11} \mathrm{~N}: 145.1\left[(\mathrm{M}+\mathrm{H})^{+}\right]$. Found: 146.3. Anal. Calcd for $\mathrm{C}_{10} \mathrm{H}_{11} \mathrm{~N}$ : C, 82.72; H, 7.64; N, 9.65. Found: C, 82.81; H, 7.69; N, 9.53 . 


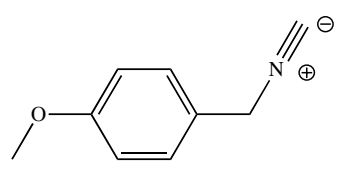

7

$7^{1}: 90 \%$ yield. Bp $93-95^{\circ} \mathrm{C}(0.05 \mathrm{~mm}) .{ }^{5} \mathrm{H}$ NMR $\left(\mathrm{CDCl}_{3}\right): \delta(\mathrm{ppm}) 7.28-7.12(\mathrm{~m}$, $2 \mathrm{H}), 6.82-6.77(\mathrm{~m}, 2 \mathrm{H}), 4.61(\mathrm{~m}, 2 \mathrm{H}), 3.72(\mathrm{~s}, 3 \mathrm{H}) .{ }^{13} \mathrm{C} \mathrm{NMR}\left(\mathrm{CDCl}_{3}\right): \delta(\mathrm{ppm}) 163.9$, 161.0, 131.9, 130.1, 114.2, 55.5, 46.2. IR (neat) 3038, 3004, 2956, 2932, 2149, 1666, 1513, 1249, 818. Anal. Calcd for $\mathrm{C}_{9} \mathrm{H}_{9} \mathrm{NO}: \mathrm{C}, 73.45 ; \mathrm{H}, 6.16 ; \mathrm{N}, 9.52$. Found: C, $73.41 ; \mathrm{H}, 6.25 ; \mathrm{N}, 9.43$.

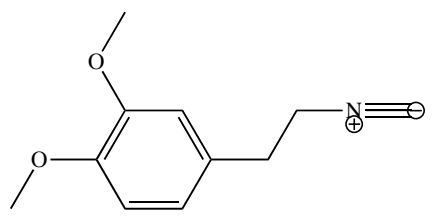

8

8: $90 \%$ yield. IR: (neat) 2999, 2924, 2850, 2147, 1674, 1590, 1516, 1261, 1025. ${ }^{1} \mathrm{H}$ $\operatorname{NMR}\left(\mathrm{CDCl}_{3}\right): \delta(\mathrm{ppm}) 6.72(\mathrm{~m}, 3 \mathrm{H}), 3.84(\mathrm{~s}, 6 \mathrm{H}), 3.63(\mathrm{t}, \mathrm{J}=7 \mathrm{~Hz}, 2 \mathrm{H}), 2.71(\mathrm{t}, \mathrm{J}=$ $7 \mathrm{~Hz}, 2 \mathrm{H}) .{ }^{13} \mathrm{C} \mathrm{NMR}\left(\mathrm{CDCl}_{3}\right): \delta(\mathrm{ppm}) 156.5,150.1,146.5,132.9,122.2,115.7$, 113.1, 56.8, 56.1, 45.7, 37.1. Anal. Calcd for $\mathrm{C}_{11} \mathrm{H}_{13} \mathrm{NO}_{2}$ : C, 69.09; H, 6.85; N, 7.32. Found: C, 69.01; H, 6.89; N, 7.24.

(5) 90-95 C (0.05 mm) (Ugi, I.; Fetzer, U.; Eholzer, U.; Knupfer, H.; Offermann, K. Angew. Chem. Int. Ed. Engl. 1965, 4, 472). 


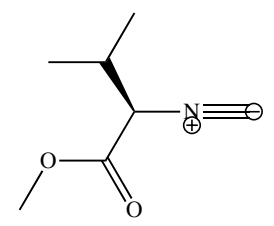

9

9: $[\alpha]^{20}{ }_{D}+5.36\left(c 1.7, \mathrm{CHCl}_{3}\right),\left[\right.$ lit. $^{6}[\alpha]^{20}{ }_{\mathrm{D}}+5.5$ (c 3.4, $\left.\mathrm{CHCl}_{3}\right]$; IR (neat) 2985, 2145, 1750. ${ }^{1} \mathrm{H}$ NMR $\left(\mathrm{CDCl}_{3}\right): \delta(\mathrm{ppm}) 4.27(\mathrm{~d}, 1 \mathrm{H}), 3.93(\mathrm{~s}, 3 \mathrm{H}), 2.03-2.74(\mathrm{~m}, 1 \mathrm{H}), 1.09$ $(\mathrm{dd}, 6 \mathrm{H}) .{ }^{13} \mathrm{C} \mathrm{NMR}\left(\mathrm{CDCl}_{3}\right): \delta(\mathrm{ppm}) 172.5,160.8,62.8,53.2,26.4,18.9$. Anal. Calcd for $\mathrm{C}_{7} \mathrm{H}_{11} \mathrm{NO}_{2}$ : C, 59.56; H, 7.85; N, 9.92. Found: C, 59.50; H, 7.90; N, 9.81.

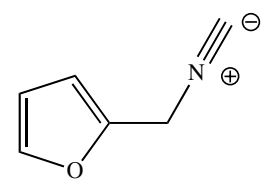

10

10: $86 \%$ yield. Bp $85-87^{\circ} \mathrm{C}(50 \mathrm{~mm})$. IR (neat): 2130 (NC), 1745. ${ }^{1} \mathrm{H}$ NMR $\left(\mathrm{CDCl}_{3}\right)$ : $\delta(\mathrm{ppm}) 7.40(\mathrm{~m}, 1 \mathrm{H}), 6.31(\mathrm{~m}, 1 \mathrm{H}), 5.90(\mathrm{~s}, 1 \mathrm{H}), 4.45(\mathrm{~s}, 2 \mathrm{H}) .{ }^{13} \mathrm{C}$ NMR $\left(\mathrm{CDCl}_{3}\right)$ : $\delta(\mathrm{ppm}) 161.4,151.1,142.1,110.3,107.4,44.5$. Anal. Calcd for $\mathrm{C}_{6} \mathrm{H}_{5} \mathrm{NO}$ : C, 67.28; H, 4.71; N, 13.08. Found: C, 67.35; H, 4.79; N, 13.01. 


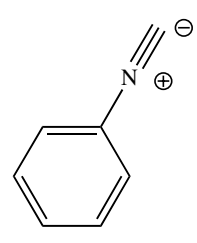

11

11: $1190 \%$ yield. Bp $50-52^{\circ} \mathrm{C}(11 \mathrm{~mm}) .{ }^{7}{ }^{1} \mathrm{H}$ NMR $\left(\mathrm{CDCl}_{3}\right): \delta(\mathrm{ppm}) \quad 7.40-7.32(\mathrm{~m}$, 2H), 7.20-7.08 (m, 3H). Anal. Calcd for $\mathrm{C}_{7} \mathrm{H}_{5} \mathrm{~N}: \mathrm{C}, 81.53 ; \mathrm{H}, 4.89 ; \mathrm{N}, 13.58$. Found: C, 81.60; H, 4.92; N, 13.44 .

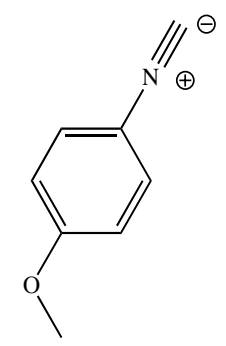

12

12: $75 \%$ yield. Bp $114-116^{\circ}(5 \mathrm{~mm}), \mathrm{mp} 30^{\circ} \mathrm{C} .^{8}$ IR (neat) $3051,2988,2123,1616$, $1635,1613,1487,1245,1111,1028 .{ }^{1} \mathrm{H}$ NMR $\left(\mathrm{CDCl}_{3}\right): \delta(\mathrm{ppm}) \quad$ 7.55-7.52 (ad, $\left.2 \mathrm{H}\right)$, 6.90-6.87 (ad, 2H), $3.82(\mathrm{~s}, 3 \mathrm{H}) .{ }^{13} \mathrm{C}$ NMR $\left(\mathrm{CDCl}_{3}\right): \delta(\mathrm{ppm}) \quad 166.6,163.3,129.0$, $122.9,116.3,55.7$

(7) Bp: 50-52 (11 mm). Weber, W. P.; Gokel, G Tetrahedron Lett. 1972, 1637.

(8) Hammick, D. L.; New, R. C. A.; Sidgwick, N. V.; Sutton, L. E. J. Chem, Soc 1930, 1876. 


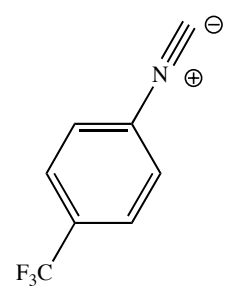

13

13: $90 \%$ yield. IR (neat) $2148(\mathrm{NC}) .{ }^{1} \mathrm{H}$ NMR $\left(\mathrm{CDCl}_{3}\right): \delta(\mathrm{ppm})$ 7.95-7.92 (ad, $\left.2 \mathrm{H}\right)$, 7.50-7.47 (ad, 2H). ${ }^{13} \mathrm{C} \mathrm{NMR}\left(\mathrm{CDCl}_{3}\right): \delta(\mathrm{ppm}) 163.2,140.6,127.8,126.4,119.7$. 118.1. Anal. Calcd for $\mathrm{C}_{8} \mathrm{H}_{4} \mathrm{~F}_{3} \mathrm{~N}$ : C, 56.15; H, 2.36; N, 8.19. Found: C, 56.22; H, 2.43; N, 8.09.

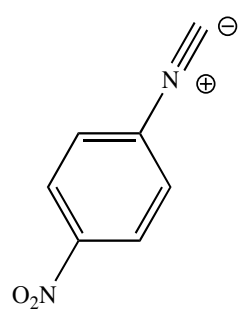

14

14: $92 \%$ yield. IR (neat): $2143 .{ }^{1} \mathrm{H}$ NMR $\left(\mathrm{CDCl}_{3}\right): \delta(\mathrm{ppm}) 8.3(\mathrm{~d}, 2 \mathrm{H}), 6.78(\mathrm{~d}, 2 \mathrm{H})$. ${ }^{13} \mathrm{C}$ NMR $\left(\mathrm{CDCl}_{3}\right): \delta(\mathrm{ppm})$ 164.7, 155.8, 135.3, 130.1, 122.5. Anal. Calcd for $\mathrm{C}_{7} \mathrm{H}_{4} \mathrm{~N}_{2} \mathrm{O}_{2}$ : C, 56.76; H, 2.72; N, 18.91. Found: C, 56.62; H, 2.83; N, 18.88. 


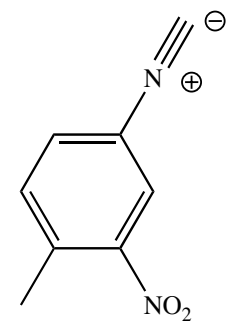

15

15: $90 \%$ yield. ${ }^{1} \mathrm{H}$ NMR $\left(\mathrm{CDCl}_{3}\right): \delta(\mathrm{ppm}) 8.48(\mathrm{as}, 1 \mathrm{H}), 7.80-7.74(\mathrm{ad}, 2 \mathrm{H}), 7.23-$ $7.20(\mathrm{ad}, 2 \mathrm{H}), 2.49(\mathrm{~s}, 3 \mathrm{H}) .{ }^{13} \mathrm{C} \mathrm{NMR}\left(\mathrm{CDCl}_{3}\right): \delta(\mathrm{ppm})$ 163.3, 148.6, 137.3, 132.3, 127.5, 124.6, 116.4, 13.5. Anal. Calcd for $\mathrm{C}_{8} \mathrm{H}_{6} \mathrm{~N}_{2} \mathrm{O}_{2}$ : C, 59.26; H, 3.73; N, 17.28. Found: C, 59.14; H, 3.79; N, 19.81. 